\title{
Abell 1451 and 1RXS J131423.6-251521: A multi-wavelength study of two dynamically perturbed clusters of galaxies ${ }^{\star}$
}

\author{
I. Valtchanov ${ }^{1}$, T. Murphy ${ }^{2, \star \star}$, M. Pierre ${ }^{1}$, R. Hunstead ${ }^{2}$, and L. Lémonon ${ }^{1}$ \\ ${ }^{1}$ CEA/DSM/DAPNIA, Service d'Astrophysique, 91191 Gif-sur-Yvette, France \\ 2 School of Physics, University of Sydney, NSW 2006, Australia \\ Received 9 April 2002 / Accepted 20 June 2002

\begin{abstract}
We present results from optical, X-ray and radio observations of two X-ray bright $\left(L_{\mathrm{X}} \sim 10^{45} \mathrm{erg} \mathrm{s}^{-1}\right)$ galaxy clusters. Abell 1451 is at redshift $z=0.1989$ and has line-of-sight velocity dispersion $\sigma_{\mathrm{v}}=1330 \mathrm{~km} \mathrm{~s}^{-1}$ as measured from 57 cluster galaxies. It has regular X-ray emission without signs of substructure, a Gaussian velocity distribution, lack of a cooling flow region and significant deviations from the observed scaling laws between luminosity, temperature and velocity dispersion, indicating a possible merging shock. There is only one spectroscopically confirmed cluster radio galaxy, which is close to the X-ray peak.

1RXS J131423.6-251521 (for short RXJ1314-25) has $z=0.2474$ and $\sigma_{\mathrm{v}}=1100 \mathrm{~km} \mathrm{~s}^{-1}$ from 37 galaxies. There are two distinct galaxy groups with a projected separation of $\approx 700 \mathrm{kpc}$. The velocity histogram is bi-modal with a redshift-space separation of $\sim 1700 \mathrm{~km} \mathrm{~s}^{-1}$, and the X-ray emission is double peaked. Although there are no spectroscopically confirmed cluster radio galaxies, we have identified a plausible relic source candidate.
\end{abstract}

Key words. galaxies: clusters: individual: Abell 1451, 1RXS J131423.6-251521 - X-rays: galaxies - radio lines: galaxies galaxies: distances and redshifts - galaxies: clusters: general

\section{Introduction}

Clusters of galaxies form a representative population which traces the highest initial density fluctuation peaks. They are excellent tools for exploring the distant Universe and are used to constrain cosmological models. A significant fraction of clusters shows evidence of substructure (e.g. Geller \& Beers 1982; Dressler \& Shectman 1988; West 1994; for a recent review see Pierre \& Starck 1998) and complexity in the distribution of the different constituents - galaxies, gas, dark matter (Baier et al. 1996). Combined multi-wavelength observations are needed to disentangle the dynamical state of clusters. Analysis of the velocity and space distribution of the galaxies is very important but, in contrast to the optical, the X-ray analysis is less prone to projection effects and probes better the cluster mass distribution (because the X-ray surface brightness depends on the square of the matter density). The presence of substructure is also revealed in the radio properties of clusters (Röttgering et al. 1994; Reid et al. 1998), but the radio sources in turn may also influence the X-ray emission (Rizza et al. 2000).

Send offprint requests to: I. Valtchanov,

e-mail: ivaltchanov@cea.fr

^ Tables ?? and ?? and Figs. ?? and ?? are only available in electronic form at http://www. edpsciences.org

$\star \star$ Now at Institute of Astronomy, University of Edinburgh, Royal Observatory, Blackford Hill, Edinburgh, EH9 3HJ.
This paper is the third in a series of papers (Pierre et al. 1997; Lémonon et al. 1997) dedicated to studies of distant, bright X-ray clusters discovered in the ROSAT All-Sky Survey (RASS, Voges et al. 1999). A sample of $\approx 10$ clusters with $L_{X}>10^{44} \mathrm{erg} \mathrm{s}^{-1}$ was selected (Pierre et al. 1994) in the redshift range $0.1<z<0.3$. In this paper we present multi-wavelength observations of two of these clusters - Abell 1451 and 1RXS J131423.6-251521 (hereafter shortened to RXJ1314-25). General data associated with both clusters are given in Table 1.

The plan of the paper is as follows: in Sect. 2 we present optical observations, data reduction, redshift catalogues and data analysis for the two clusters. In Sects. 3 and 4 we present ROSAT-HRI observations and data analysis and ATCA radio observations and data analysis respectively. Finally in Sect. 5 we discuss the multi-wavelength view of Abell 1451 and RXJ1314-25. Throughout the paper we use $H_{0}=50 \mathrm{~km} \mathrm{~s}^{-1} \mathrm{Mpc}^{-1}$ and $q_{0}=0.5$.

\section{Optical observations, data reduction and analysis}

\subsection{Observations and reduction}

Multi-Object Slit (MOS) spectra for both clusters were acquired using the ESO 3.6-m telescope at La Silla in two runs, one in 1993 and one in 1999 (Table 2). The first run 
Table 1. Properties of the two clusters. References: Abell (1958), Abell et al. (1989) for the coordinates and richness; Bautz \& Morgan (1970) for classification for Abell 1451; coordinates for RXJ1314-25 are from RASS (Voges et al. 1999); $T_{\mathrm{X}}$ and $L_{\mathrm{X}}$ in the $2-10 \mathrm{keV}$ band are from Matsumoto et al. (2001); redshift and $\sigma_{\mathrm{v}}$ are from this paper; the apparent magnitude of an $L^{*}$ galaxy was calculated using $M^{*}\left(B_{j}\right)=-21.8$, obtained by adjusting the Lumsden et al. (1997) value of $M^{*}\left(B_{j}\right)=-20.16$ to the cosmological parameters used in this paper.

\begin{tabular}{lcc}
\hline \hline & Abell 1451 & RXJ1314-25 \\
\hline RA (J2000) & $12: 03: 16.0$ & $13: 14: 23.6$ \\
Dec (J2000) & $-21: 30: 42$ & $-25: 15: 21$ \\
BM Class & III & - \\
Richness & 3 & - \\
Redshift & 0.1989 & 0.2474 \\
$\sigma_{\mathrm{v}} \mathrm{km} \mathrm{s}^{-1}$ & 1330 & 1100 \\
$B_{j}^{*}$ & 19.5 & 20.2 \\
$L_{\mathrm{X}}\left(10^{45}\right) \mathrm{erg} \mathrm{s}^{-1}$ & 1.5 & 1.8 \\
$T_{\mathrm{X}} \mathrm{keV}$ & 13.4 & 8.7 \\
\hline
\end{tabular}

was equipped with EFOSC1 (ESO Faint Object Spectrograph and Camera), Tektronix $512 \times 512 \mathrm{CCD}$ (pixel size $27 \mu \mathrm{m}$ and $0.61^{\prime \prime} /$ pixel) and Grism B300, covering the range 3740-6950 А, with central wavelength $5250 \AA$ and dispersion of $6.2 \AA /$ pixel. The observations in 1999 were performed with EFOSC2, CCD \#40 $2048 \times 2048$ (pixel size $15 \mu \mathrm{m}$, $0.157^{\prime \prime} /$ pixel) and Grism O300 which covers 3860-8070 with central wavelength $5000 \AA$ and dispersion $2.06 \AA$ /pixel. However, the final wavelength range depends on the position of each slit on the mask. The seeing was 1.0-1.2"for both 1993 and 1999 runs. The corresponding airmasses and the exposure times for each mask/slit configuration are given in Table 2.

All spectra were processed within the ESO-MIDAS or IRAF environment to produce the final wavelength calibrated, sky subtracted spectra. A HeAr lamp was used for wavelength calibration, and a second-order cubic spline or polynomial fit to 5-12 comparison lines gave rms residuals $<1 \AA$.

We have determined redshifts using the Tonry \& Davis (1979) cross-correlation technique as implemented in the RVSAO package (Kurtz \& Mink 1998). Before processing with RVSAO we have masked out spectral ranges where significant residuals from sky subtraction could occur, and also any possible emission lines. Generally we have cross-correlated our observed spectra with 3 to 6 galaxy and star templates with good signal-to-noise, and also with the composite absorption-line template (fabtemp97) distributed with RVSAO. For each target object we adopted the redshift value from the best (highest correlation coefficient) template. The redshifts were then checked by identifying the prominent absorption features (CaII $H \& K$, $G$ band, $\mathrm{MgI}$ triplet) in each spectrum. If emission lines were present in the spectra, redshifts were determined from them.

The errors in redshift were computed from $\Delta z=k /(1+r)$, where $r$ is the cross-correlation coefficient and $k$ was determined empirically by adding noise to a high signal-to-noise spectrum and correlating it with template spectra with known velocities. For our observational configurations we found $k=0.003$, which gives a velocity error of about $200 \mathrm{~km} \mathrm{~s}^{-1}$ for a redshift estimate with $r=3$. We have checked our redshift measurements and uncertainties using a few galaxy spectra repeated in different runs or masks and they are all in excellent agreement, consistent with the derived errors.

We have not converted our measurements to the heliocentric system because the correction is about $1 \mathrm{~km} \mathrm{~s}^{-1}$ for the 1993 run and $-8.8 \mathrm{~km} \mathrm{~s}^{-1}$ for the 1999 run, well below the uncertainties.

The final redshift catalogue of objects for Abell 1451 is shown in Table ?? and for RXJ1314-25 in Table ??, with corresponding finding charts in Figs. 1 and 3.

\subsection{Optical data analysis}

\subsubsection{Abell 1451}

Cluster membership was determined by using an interactive version of the Beers et al. (1990) ROSTAT package (Valtchanov 1999) and confirmed by the 3- $\sigma$ clipping method of Yahil \& Vidal (1977). The cluster redshift distribution is shown in Fig. 4 and some relevant characteristics, taking redshift errors into account, are given in Table 5. The distribution is very close to Gaussian, which has been quantified by various statistical tests for normality (D'Agostino \& Stephens 1986) the Wilk-Shapiro test accepts normality at the $99 \%$ level and the Anderson-Darling test at the $95 \%$ level.

There is no single dominant cluster galaxy. The brightest cluster members (BCMs, \#34, \#39, \#36 and \#40) are linearly aligned in projection (see Fig. 2) and their spectra are typical elliptical galaxy absorption spectra with no emission lines. The projected galaxy density distribution from SuperCOSMOS (Hambly et al. 2001) data shows an elongation in the same direction. The velocity dispersion among the BCMs is $\sim 800 \mathrm{~km} \mathrm{~s}^{-1}$, somewhat less than the overall cluster velocity dispersion.

\subsubsection{RXJ1314-25}

Statistics of the redshift distribution are shown in Table 6 and the redshift histogram in Fig. 5. The distribution clearly shows two peaks and the test for the hypothesis of a unimodal distribution - the dip test (Hartigan 1985) - gives an insignificant probability of a single mode. Going further, we have applied the KMM method for detecting bimodality (Ashman et al. 1994), which confirmed the dip test negative result for unimodality and also gave us the probable group membership, assuming a bi-modal distribution. In Table 6 we show the same statistics for both groups. Although the number of cluster members is relatively small for giving high weight to the KMM result, the segregation of the galaxies on the sky supports that conclusion (see Fig. 6).

The optical image of the cluster (Fig. 3) shows two dominant galaxies (\#48 and \#19, marked also in Table ??) as the brightest cluster galaxies (BCG). Both have typical giant elliptical galaxy absorption line spectra, without any apparent emission lines. The second-ranked BCG $(\# 19, z=0.2503)$ is at rest with respect to the galaxy members of the western 


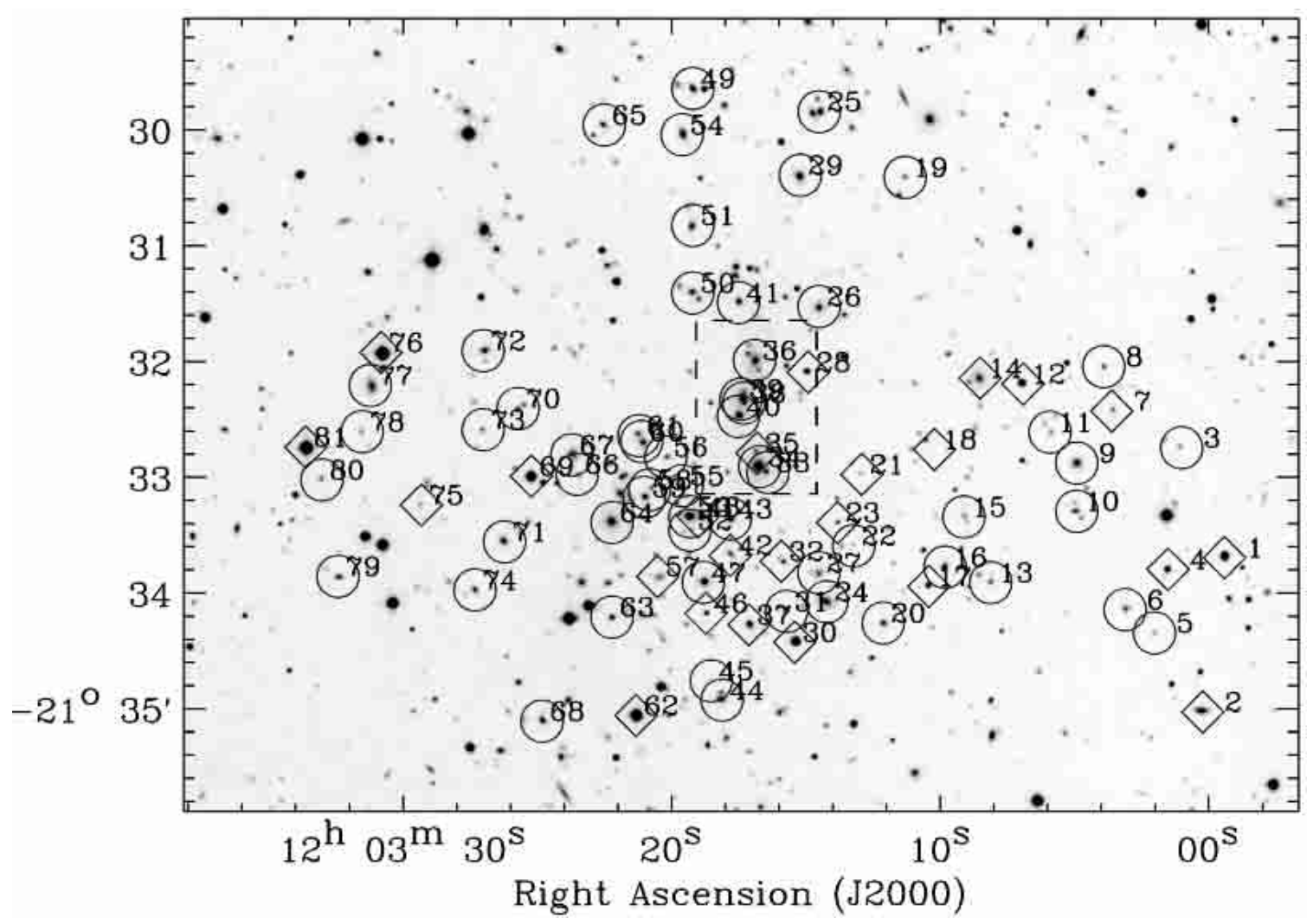

Fig. 1. Abell 1451: $V$-band image from the Danish 1.5-m telescope showing the objects included in the spectroscopic study. Numbers correspond to the object identification in Table ??; those denoted as diamonds are either stars or non-cluster members. The dashed box is the central part, shown zoomed in Fig. 2.

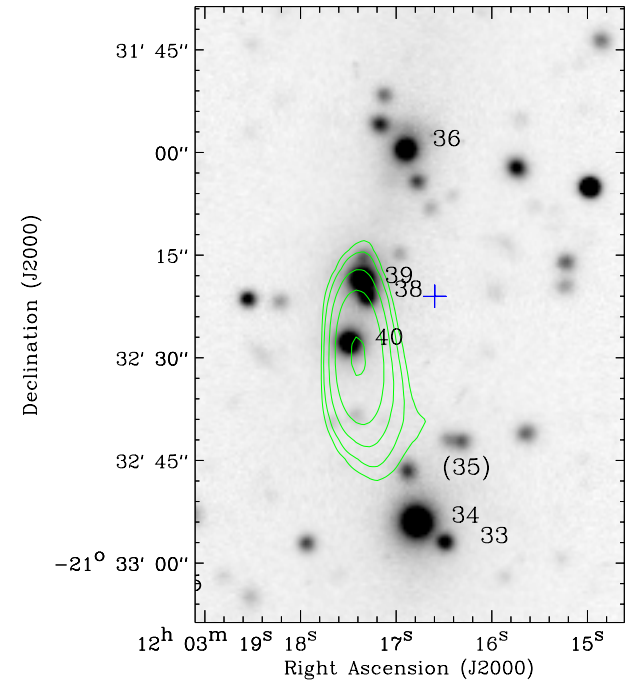

Fig. 2. Abell 1451: zoomed image of the central part of Fig. 1. The plus sign marks the X-ray emission centre (see Sect. 3) and the contours are ATCA $13 \mathrm{~cm}$ radio observation with levels $=0.3,0.5,1,2$, 5 and $10 \mathrm{mJy} / \mathrm{beam}$, rms noise level is $60 \mu \mathrm{Jy} / \mathrm{beam}$ (see Sect. 4). The object in brackets is a background galaxy.

group, while the first-ranked galaxy (\#48, $z=0.2463$ ) differs by $\sim 800 \mathrm{~km} \mathrm{~s}^{-1}$ from the mean redshift of the eastern group, but lies closer to the overall cluster mean. The separation between the two groups in velocity space is $\sim 1700 \mathrm{~km} \mathrm{~s}^{-1}$, substantially greater than their combined velocity dispersions. With optical data alone we can not determine whether the two groups are gravitationally connected.

\section{X-ray data and analysis}

The X-ray observing logs for both clusters are given in Table 7 . For both ROSAT/HRI images we have used 5"/pixel binning of the event list in order to reduce the noise as much as possible without losing information on the cluster extension. The raw photon images were then filtered using wavelet analysis with Poisson noise modeling (Starck \& Pierre 1998) at $10^{-4}(\sim 4 \sigma)$ significance level for the wavelet coefficients.

\subsection{X-ray morphology}

The X-ray contours are shown overlaid on optical images and radio observations (see Sect. 4) in Fig. 7 for Abell 1451 and in Fig. 8 for RXJ1314-25.

\subsubsection{Abell 1451}

The X-ray image in Fig. 7 shows a very strong point source $\sim 1^{\prime} 30^{\prime \prime}$ south of the cluster centre which coincides with a QSO at $z=1.17$ (object \#32 in Table ??). The cluster emission is regular, with the inner contours slightly twisted but no sign of substructure. The X-ray emission peaks at RA $=12^{\mathrm{h}} 03^{\mathrm{m}} 16^{\mathrm{s}} .6$ and Dec $=-21^{\circ} 32^{\prime} 21^{\prime \prime}$, which is $36^{\prime \prime}(150 \mathrm{kpc})$ north of the 
Table 2. Summary of the multi-slit spectroscopic observations.

\begin{tabular}{|c|c|c|c|c|c|}
\hline \multirow[t]{2}{*}{ Date } & Object & RA Dec & Airmass & MOS & Exposure \\
\hline & \multicolumn{3}{|c|}{ (J2000) } & masks/slits & (s) \\
\hline 1993 Mar. 29 & Abell 1451 & $12: 03: 15-21: 31: 36$ & 1.2 & $1 / 15$ & 900 \\
\hline 1993 Mar. 29 & RXJ1314-25 & $13: 14: 29-25: 16: 25$ & 1.1 & $1 / 15$ & $2 \times 900+1800$ \\
\hline 1999 Apr. 19/20 & Abell 1451E & $12: 03: 12-21: 33: 25$ & 1.34 & $2 / 20+21$ & $3 \times 1800 / 4 \times 2100$ \\
\hline 1999 Apr. 19/20 & Abell 1451W & $12: 03: 23-21: 33: 25$ & 1.40 & $2 / 21+19$ & $2 \times 3600 / 4200$ \\
\hline 1999 Apr. 19/20 & RXJ1314-25N & $13: 14: 22-25: 14: 55$ & 1.06 & $2 / 17+18$ & $2 \times 3600 / 4 \times 2100$ \\
\hline 1999 Apr. 19/20 & RXJ1314-25S & $13: 14: 22-25: 17: 55$ & 1.04 & $2 / 17+18$ & $4 \times 1800 / 4 \times 2100$ \\
\hline
\end{tabular}

Table 5. Abell 1451: some statistical characteristics of the redshift distribution, corrected for measurement errors (Danese et al. 1980). The scale measure is in the cluster rest frame, i.e., $S_{\mathrm{BI}}=c \sigma_{z} /(1+z)$ (Harrison 1974). The errors $(1 \sigma)$ are calculated by using the accelerated bias-corrected bootstrap technique with 1000 bootstrap resamplings (Efron \& Tibshirani 1986).

\begin{tabular}{ll}
\hline \hline \multicolumn{1}{c}{ Characteristic } & \multicolumn{1}{c}{ Value } \\
\hline \multicolumn{1}{c}{$N=57$} & \\
Bi-weighted location: $C_{\mathrm{BI}}$ & $\bar{z}=0.1989_{-0.0007}^{+0.0005}$ \\
Bi-weighted scale: $S_{\mathrm{BI}}$ & $1330_{-90}^{+130} \mathrm{~km} \mathrm{~s}^{-1}$ \\
Maximum gap & $564 \mathrm{~km} \mathrm{~s}^{-1}$ \\
\hline
\end{tabular}

brightest cluster galaxy (\#34 in Table ??) and 12" west of the second brightest galaxy (\#39), and $\sim 1^{\prime} 40^{\prime \prime}$ from the catalogued cluster position (Abell et al. 1989).

\subsubsection{RXJ1314-25}

The cluster X-ray emission is quite irregular, showing two central peaks and a SE-NW elongation. Unfortunately, there is an X-ray emitting star projected in front of the cluster (object \#29 in Table ??) only $\sim 24^{\prime \prime}$ from the adopted X-ray centre. The strong point source SE of the cluster centre is a Sy1 galaxy (object \#47 in Table ??) and a cluster member. There is no indication of substructure in the X-ray emission associated with the eastern group or the brightest cluster galaxy (see Fig. 6).

\section{2. $X$-ray properties}

To estimate the basic physical cluster parameters we model the $\mathrm{X}$-ray surface brightness using the isothermal $\beta$-model (King 1962; Cavaliere \& Fusco-Femiano 1976)

$S(r)=S_{0}\left(1+\left(r / r_{\mathrm{c}}\right)^{2}\right)^{0.5-3 \beta}+S_{\mathrm{b}}$,

where $S(r)$ is the azimuthally averaged X-ray surface brightness as a function of the radial distance $r$ from the centre, $S_{0}$ is the central brightness, $S_{\mathrm{b}}$ is the background contribution and $r_{\mathrm{c}}$ is the core radius. To fit the model profile to the data we define a proper cluster centre and exclude any discrete sources projected over the cluster X-ray emission. Finally, we obtain an average profile by summing the cluster X-ray photons in concentric rings and fit the model (Eq. (1)) with $S_{0}, S_{\mathrm{b}}, \beta$ and $r_{\mathrm{c}}$ as free parameters, taking into account the Poissonian errors of the rings counts. The X-ray surface brightness profiles of Abell 1451 and RXJ1314-25 are shown in Figs. 9, 10, with the corresponding parameters in Table 8 .

To derive the count-rate in the $[0.1-2.4] \mathrm{keV}$ ROSAT/HRI band we integrate the fitted surface brightness profile analytically, excluding the background. The integration is usually carried out to a given radius $r_{\mathrm{lim}}$, where the surface brightness profile reaches the detection limit; for both clusters we put $r_{\lim }=300^{\prime \prime}$. For the overall count rate $C$ we have

$$
\begin{aligned}
C\left(<r_{\lim }\right)= & \int_{0}^{r_{\lim }} 2 \pi r S(r) \mathrm{d} r=\frac{\pi S_{0} r_{\mathrm{c}}^{2}}{3 / 2-3 \beta} \\
& \times\left[\left(1+\left(r_{\lim } / r_{\mathrm{c}}\right)^{2}\right)^{3 / 2-3 \beta}-1\right] .
\end{aligned}
$$

By means of EXSAS (Zimmermann et al. 1994), the observed count-rate is then used to normalize the spectral model a Raymond \& Smith (1977) thermal plasma emission, convolved by the ROSAT/HRI response function, with temperature and metallicity from ASCA data (Matsumoto et al. 2001) and line-of-sight Galactic absorption by HI from Dickey \& Lockman (1990). The spectrum is then integrated to derive the flux and cluster rest frame luminosity in the "standard" X-ray bands [0.5-2] and [2-10] keV. The profile model, together with the emission model, was used to derive the emission measure, $\int_{V} n_{\mathrm{e}} n_{\mathrm{p}} \mathrm{d} V$, and the proton density distribution:

$n_{\mathrm{p}}(r)=n_{\mathrm{p}}(0)\left(1+\left(r / r_{\mathrm{c}}\right)^{2}\right)^{-3 \beta / 2}$.

Assuming a hydrogen gas then $\rho_{\text {gas }}=2.21 \mu m_{\mathrm{p}} n_{\mathrm{p}}$ and we can calculate the mass of the gas inside a given radius $R$ :

$M_{\text {gas }}(r<R)=\int_{0}^{R} 4 \pi \rho_{\text {gas }}(r) \mathrm{d} r$.

The total gravitating mass of the cluster within radius $r$ can be estimated, assuming hydrostatic equilibrium, as

$M_{\mathrm{tot}}(r)=\frac{r^{2} k T}{G \mu m_{\mathrm{p}}}\left(\frac{1}{n} \frac{\mathrm{d} n}{\mathrm{~d} r}+\frac{1}{T} \frac{\mathrm{d} T}{\mathrm{~d} r}\right)$,

with $n=n_{\mathrm{e}}+n_{\mathrm{p}}=2.21 n_{\mathrm{p}}$ and $\mu=0.61$ for the abundances. Ignoring any radial temperature dependence we can derive:

$M_{\mathrm{tot}}(r)=3 \beta r^{3} k T / G \mu m_{\mathrm{p}}\left(r^{2}+r_{c}^{2}\right)$.

The derived and model parameters for the X-ray emission of both clusters are shown in Table 9. There are small differences 




Fig. 3. RXJ1314-25: I-band image from the Danish 1.5-m telescope showing the objects in the spectroscopic study. Numbers correspond to the object identifications in Table ??; those marked with diamonds denote either stars or non-cluster members. Note that we have not plotted galaxy \#20 because it is not resolved spatially from galaxy \#19.

in luminosity when compared with ASCA data. This can be explained, firstly, by the smaller limiting distance ( $\left.4^{\prime}\right)$ for flux integration adopted in Matsumoto et al. (2001) and secondly, by the fact that for both clusters there are discrete X-ray sources within the extended cluster emission which were not resolved by ASCA and so their contribution was not subtracted.

Derived cluster masses should be considered with caution, as the combined X-ray/optical analysis tends to indicate that neither cluster has reached equilibrium.

Although the X-ray emission in both clusters is not centrally peaked, we have tried to estimate the cooling flow radius - the zone where the time for isobaric cooling is less than the age of the universe (Sarazin 1986; Fabian 1994). For any reasonable choice of the Hubble constant and gas parameters $\left(n_{\mathrm{p}}, T_{\mathrm{X}}, Z\right)$ there is no such zone.

\section{Radio observations and data analysis}

\subsection{Observations and reduction}

The Australia Telescope Compact Array (ATCA) consists of five $22 \mathrm{~m}$ antennas on a $3 \mathrm{~km}$ east-west railway track, and a sixth antenna $3 \mathrm{~km}$ from the western end of the track. Each cluster was observed in 1999 February for 12 hours with the ATCA in the $6 \mathrm{C}$ configuration, giving baselines ranging from $153 \mathrm{~m}$ to $6 \mathrm{~km}$. Simultaneous observations at $1.384 \mathrm{GHz}$ and $2.496 \mathrm{GHz}$ were made for each cluster.

The radio data were processed using standard MIRIAD (Sault et al. 1995) software and techniques. The primary flux density calibrator was PKS B1934-638, with PKS B1245-197 as the secondary phase calibrator. The data were then CLEANed and RESTORed. Table 10 lists details of the 


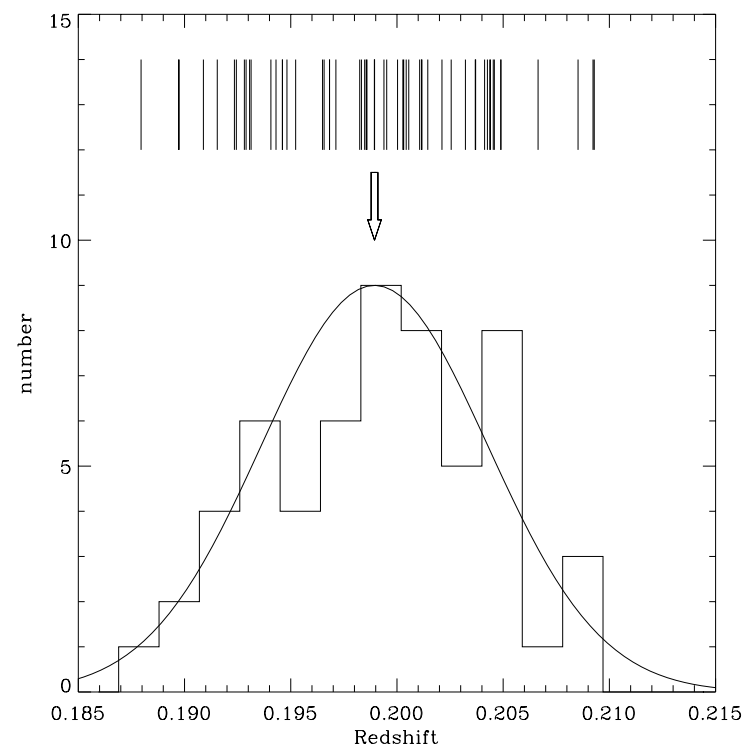

Fig. 4. Abell 1451: redshift histogram for cluster members; the bin size is 0.0019 . The bi-weighted location $\left(C_{\mathrm{BI}}\right)$ is shown by an arrow. The lines above the histogram are the actual 1-D redshift distribution and the continuous line is the Gaussian function with bi-weighted location $\left(C_{\mathrm{BI}}\right)$ and bi-weighted scale $\left(S_{\mathrm{BI}}\right)$ from Table ??.

Table 6. RXJ1314-25: statistics of the redshift distribution, as for Table 5. The subdivision into two groups follows from KMM (Ashman et al. 1994).

\begin{tabular}{lc}
\hline \hline \multicolumn{2}{c}{ Characteristic } \\
\multicolumn{1}{c}{ Total, $N=37$} \\
$C_{\mathrm{BI}}$ & $\bar{z}=0.2474_{-0.0008}^{+0.0006}$ \\
$S_{\mathrm{BI}}$ & $1100_{-90}^{+140} \mathrm{~km} \mathrm{~s}^{-1}$ \\
Maximum gap & $523 \mathrm{~km} \mathrm{~s}^{-1}$ \\
\hline \multicolumn{3}{c}{ Group 1 (East), $N=15$} \\
$C_{\mathrm{BI}}$ & $\bar{z}=0.2429_{-0.0008}^{+0.0003}$ \\
$S_{\mathrm{BI}}$ & $590_{-150}^{+110} \mathrm{~km} \mathrm{~s}^{-1}$ \\
Maximum gap & $516 \mathrm{~km} \mathrm{~s}^{-1}$ \\
\hline \multicolumn{3}{c}{ Group 2(West), $N=22$} \\
$C_{\mathrm{BI}}$ & $\bar{z}=0.2500_{-0.0005}^{+0.0006}$ \\
$S_{\mathrm{BI}}$ & $560_{-70}^{+120} \mathrm{~km} \mathrm{~s}^{-1}$ \\
Maximum gap & $523 \mathrm{~km} \mathrm{~s}^{-1}$ \\
\hline
\end{tabular}

Table 7. ROSAT-HRI X-ray observing log.

\begin{tabular}{lcc}
\hline \hline \multicolumn{1}{c}{ Cluster } & Date & Exposure (s) \\
\hline Abell 1451 & 1997 Jul. 14-16 & 25603 \\
RXJ1314-25 & 1996 Jan. 27-31 & 29294 \\
\hline
\end{tabular}

ATCA observations, including the size and orientation of the elliptical Gaussian restoring beams and the rms noise in the final images.

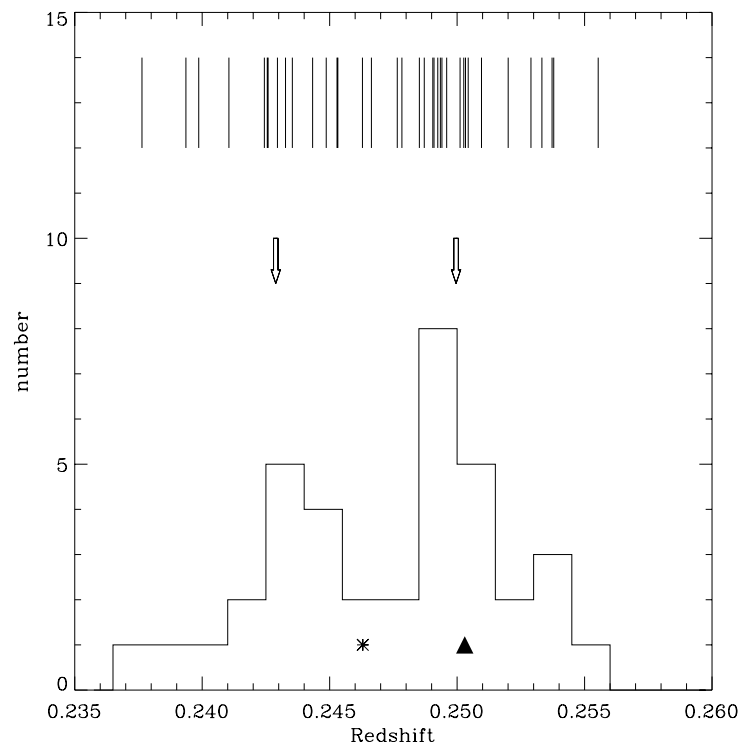

Fig. 5. RXJ1314-25: redshift histogram for cluster members; the bin size is 0.0015 . The $C_{\mathrm{BI}}$ locations for the two groups (see Table 6) are indicated by arrows. The two brightest cluster galaxies are marked: \#48 with an asterisk and \#19 with a filled triangle.

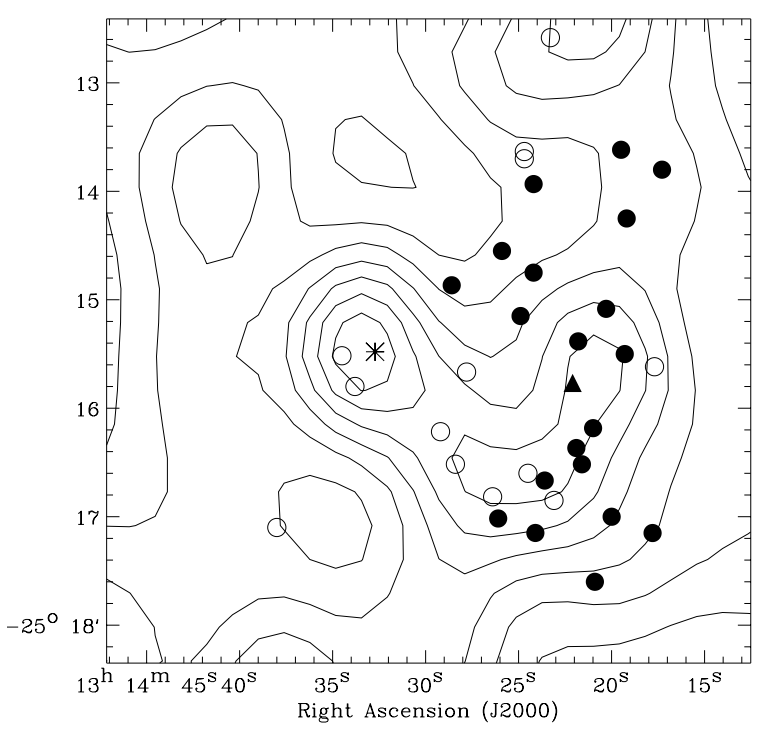

Fig. 6. RXJ1314-25: sky distribution for cluster members. Open circles denote the members of the eastern group, while the filled circles are those belonging to the western group as assigned by KMM (see Table 6). The contours are the adaptive kernel density estimate (Silverman 1986; Pisani 1996) of the SuperCOSMOS galaxy distribution. The asterisk and filled triangle mark the positions of the firstand second-ranked BCGs respectively (\#48 and \#19 in Table ??). Note that galaxy \#20 is too close to galaxy \#19 to be plotted separately.

\subsection{Data analysis}

The AIPS task VSAD was used to generate a radio source list for each cluster, above a nominal cutoff of $0.5 \mathrm{mJy}$ at $1384 \mathrm{MHz}$. An elliptical Gaussian was fitted to each source as described by Condon (1997). For sources which were extended or complex, the integrated flux density was estimated by using kview (Gooch 1996) to sum inside a rectangular region 


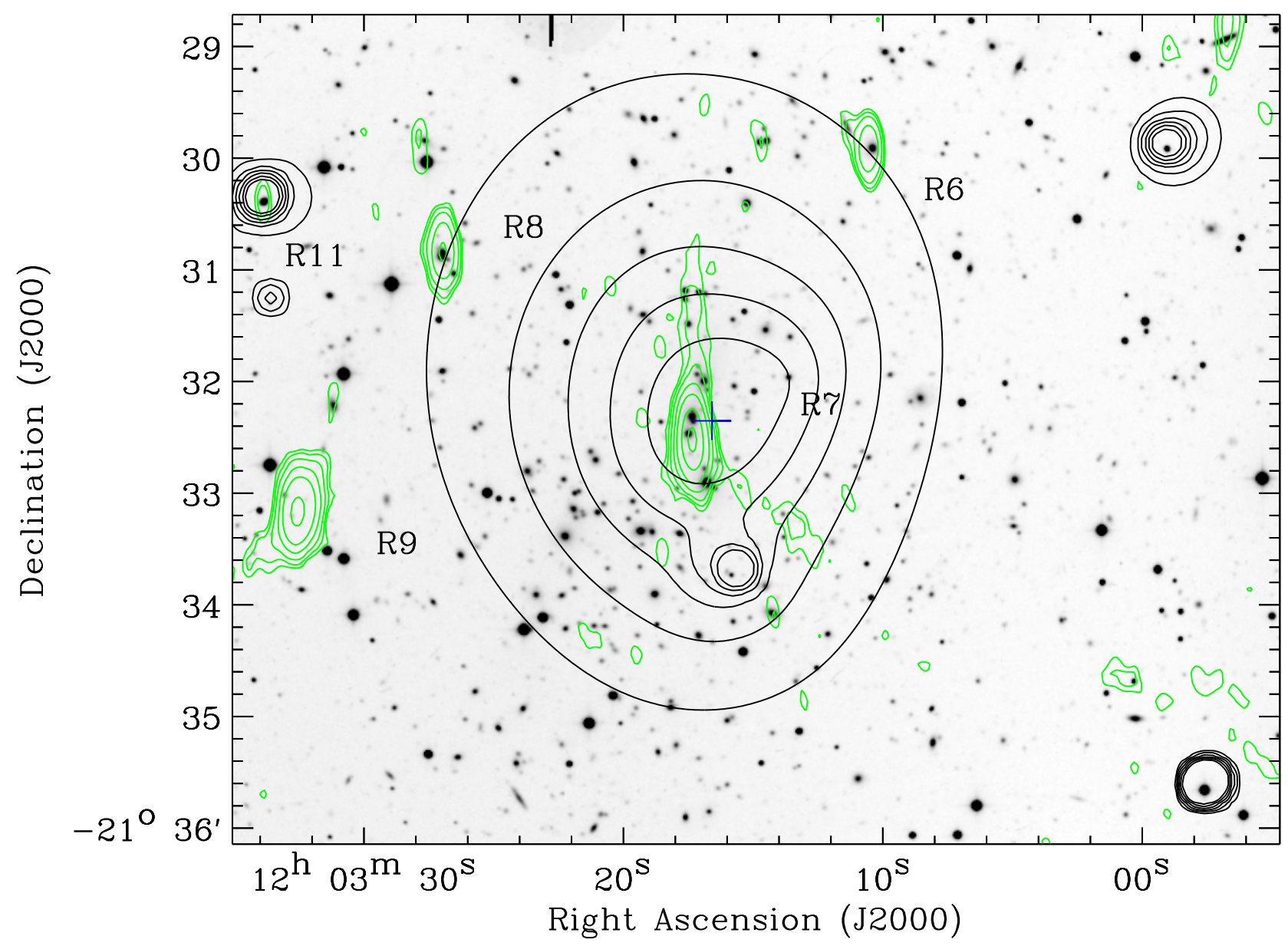

Fig. 7. Abell 1451: Optical $V$-band image from the Danish 1.5 -m telescope overlaid with HRI X-ray contours (heavy) and $20 \mathrm{~cm}$ ATCA radio contours (lightweight). The X-ray contours run from 0.006 to 0.02 counts s$^{-1} \mathrm{arcmin}^{-2}$ in logarithmic steps; the innermost cluster contour is at 0.0134 counts s $\mathrm{s}^{-1} \operatorname{arcmin}^{-2}$. The peak of the extended X-ray emission, used for the profile measurements, is marked with a cross. The radio contour levels are $0.2,0.3,0.5,1,2,5$ and $10 \mathrm{mJy} /$ beam; the rms noise level is $80 \mu \mathrm{Jy} / \mathrm{beam}$.

defined around the source. The radio sources for Abell 1451 and RXJ1314-25 within one Abell radius $\left(R_{\mathrm{A}}=1.7 / z\right.$ arcmin $)$ of the cluster centre ${ }^{1}$ are listed in Table 11 . Positions are measured from the $1384 \mathrm{MHz}$ image except for sources R1 and R9 in Abell 1451 which are measured at $2496 \mathrm{MHz}$; quoted errors are the quadratic combination of the formal VSAD error and a nominal $0.5^{\prime \prime}$ calibration uncertainty. Errors in flux density are taken directly from the VSAD output; if no error is quoted for $S_{\text {int }}$ the measurement was made using kview.

A search was carried out for optical identifications of the radio sources in Table 11 using the SuperCOSMOS catalogue (Hambly et al. 2001). A search radius of 10 arcsec was used. The results are shown in Table 12.

\subsubsection{Abell 1451}

The $1.384 \mathrm{GHz}(20 \mathrm{~cm})$ radio contours overlaid on the DSS image of the cluster field are shown in Fig. ? ${ }^{2}$; the $1.384 \mathrm{GHz}$

\footnotetext{
${ }^{1}$ For Abell 1451 we have adopted the X-ray peak as the centre of the cluster.

2 Available only in the electronic version.
}

$(20 \mathrm{~cm})$ radio contours for the central part of the cluster are shown in Fig. 7 together with the X-ray contours. The central radio source (R7 in Table 11) is extended north-south with a peak that best matches galaxy \#40 from Table ??. The apparent wide-angle tail (WAT) morphology relies on the lowest $(2.5 \sigma)$ contours and is therefore uncertain. Such morphologies are usually associated with the central dominant cluster member. An alternative interpretation, suggested by the $13 \mathrm{~cm}$ observation shown only for the central part of the cluster in Fig. 2, is that R7 is a head-tail source identified either with galaxy \#40 or the brighter galaxy \#39 which is $\sim 10^{\prime \prime}$ north. Higher resolution radio observations are needed to settle this issue.

Apart from R7, there are no other cross-identifications between the spectroscopic catalogue and radio source list. However, there are several likely cluster identifications, including the head-tail source (R1) located $\sim 8^{\prime} \mathrm{NW}$ of the cluster centre. The weakest source in the field, R11, is a clear AGN candidate - it is a strong X-ray source with a flat radio spectrum; SuperCOSMOS classifies the optical counterpart as a galaxy. R9 is an extended source with no obvious optical counterpart. We initially considered it as a possible relic source (Enßlin et al. 1998), but the $13 \mathrm{~cm}$ image suggests an identification with a 


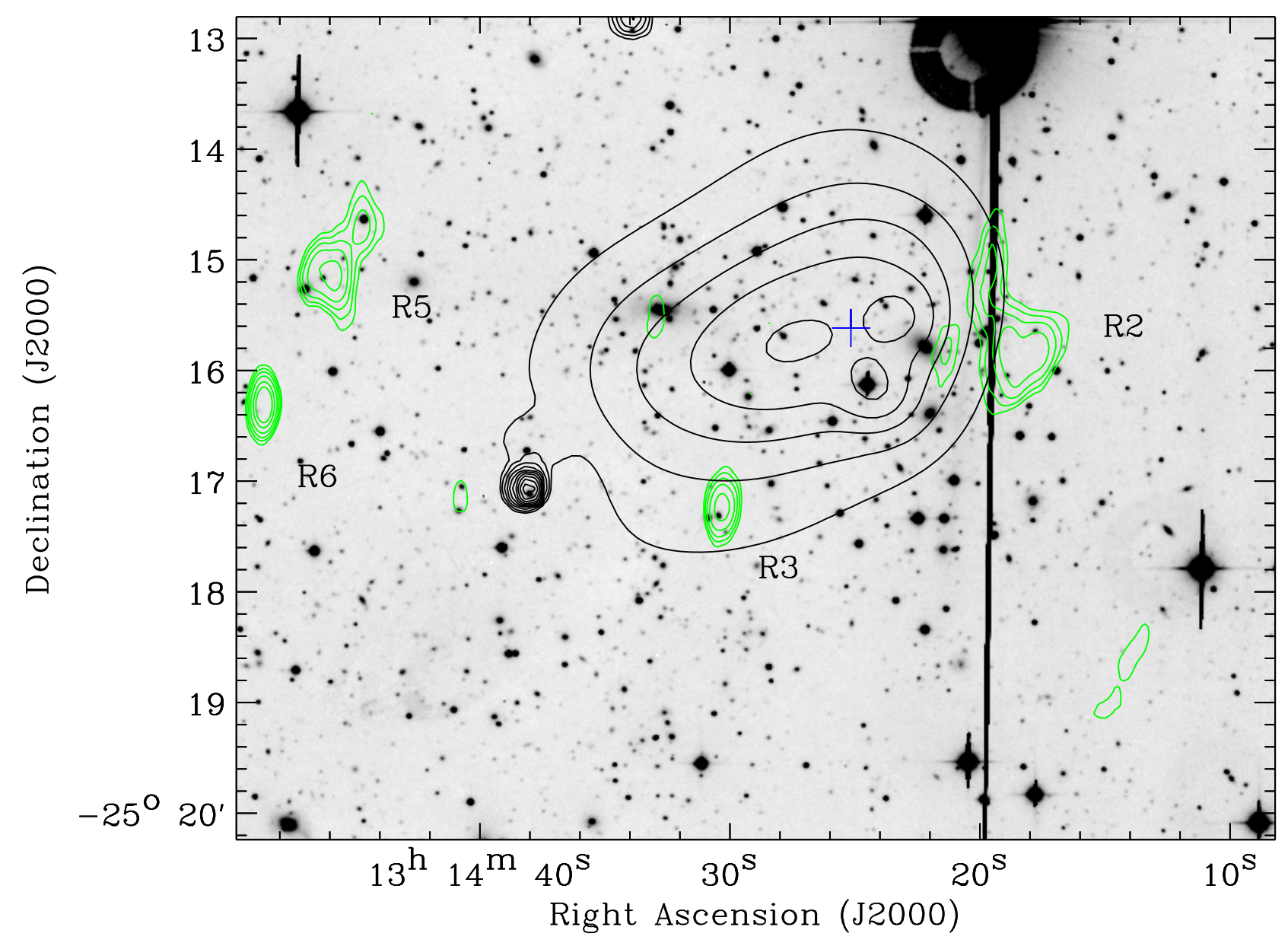

Fig. 8. RXJ1314-25: optical $I$-band image from the Danish $1.5-\mathrm{m}$ telescope overlaid with HRI X-ray contours (heavy) and $20 \mathrm{~cm}$ ATCA contours (lightweight). The X-ray contours run from 0.004 to 0.065 counts s$^{-1}$ arcmin $^{-2}$ in logarithmic steps; the innermost cluster contour is at 0.012 counts s $\mathrm{s}^{-1} \mathrm{arcmin}^{-2}$. The centre of the X-ray emission taken for the profile measurements is marked with a cross. The radio contour levels are $0.35,0.5,0.7,1,1.5$ and $2 \mathrm{mJy} / \mathrm{beam}$; the rms noise level is $90 \mu \mathrm{Jy} / \mathrm{beam}$.

very faint object visible on both the $B$ and $R$ sky survey images. The QSO $(\# 32, z=1.17)$ is radio quiet.

\subsection{2. $\mathrm{RXJ1314-25}$}

The $1.384 \mathrm{GHz}(20 \mathrm{~cm})$ radio contours overlaid on the DSS image of the cluster field are shown in Fig. ? $?^{3}$; the central region of RXJ1314-25, together with X-ray contours, are shown in Fig. 8 overlaid on the optical image from Fig. 3. Background noise in the central region of the $20 \mathrm{~cm}$ image is affected by sidelobes of the 200 mJy source NVSS J1314-2522 located $\mathrm{SW}$ of the cluster centre, just outside one Abell radius. For this reason the lowest contour level in Fig. 8 has been set at $\sim 4 \sigma$.

As shown in Table 11, the projected radio source density is lower than for Abell 1451. There are no positive identifications with spectroscopically confirmed cluster members, although there is a weak source just below the $0.5 \mathrm{mJy}$ threshold, very close to the brightest cluster galaxy BCG2 (galaxy \#48; see Fig. 8). There is also a striking mirror symmetry of sources $\mathrm{R} 2$ and R5 (Table 11) with respect to this galaxy, resembling

\footnotetext{
3 Available only in the electronic version.
}

the lobes of an FR II radio galaxy (see e.g. Fig. 8). At the redshift of the galaxy, $z=0.2466$, the linear size of $6^{\prime} 15^{\prime \prime}$ would correspond to $\sim 1 \mathrm{Mpc}$, a typical scale for a giant radio galaxy (Schoenmakers et al. 2001). Such an interpretation is questionable, however, as the galaxy is clearly not located in an underdense environment (see Fig. 6), as required for the growth of giant sources. Neither source has an obvious optical counterpart in the $20 \mathrm{~cm}$ image, but R5 appears double at $13 \mathrm{~cm}$, with a plausible optical identification midway between the components. On the other hand, with its extended radio emission, steep spectrum and position in the cluster, R5 is an excellent candidate for a relic source. Its true extent is difficult to judge from the ATCA $20 \mathrm{~cm}$ image because of sidelobe confusion, but there is a suggestion of low surface brightness, extended emission in the vicinity of BGC\#1 and the western X-ray peak.

\section{Discussion}

\subsection{Abell 1451}

The dynamical state of Abell 1451 is very similar to that of Abell 665 (Gomèz et al. 2000) and to the more distant Abell 1300 (Lémonon et al. 1997; Reid et al. 1998), suggesting 


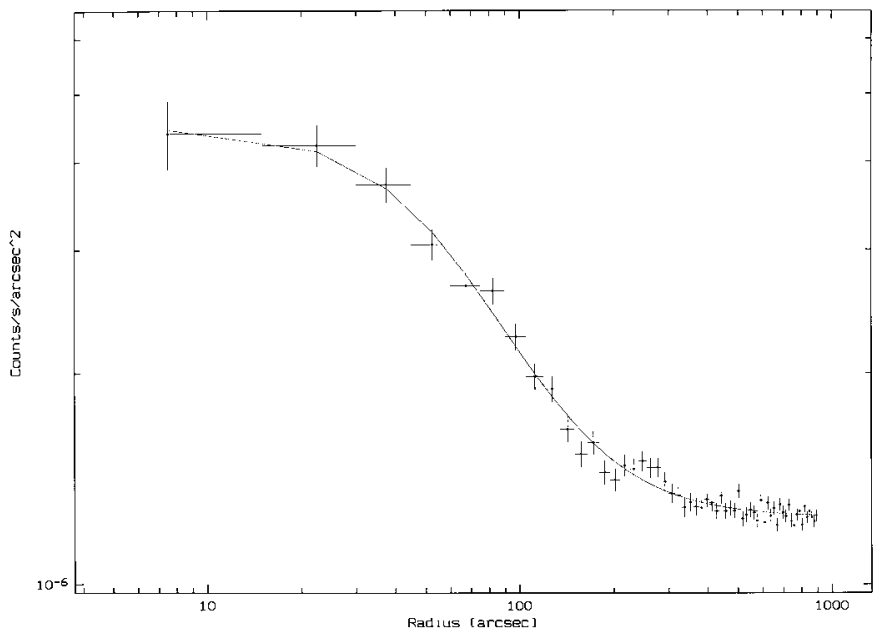

Fig. 9. Abell 1451: circularly averaged surface brightness profile in the energy band [0.1-2.4] keV. Bin size is $15^{\prime \prime}$ and the solid line is the King profile fit (Eq. (1)) with parameters in Table 8.

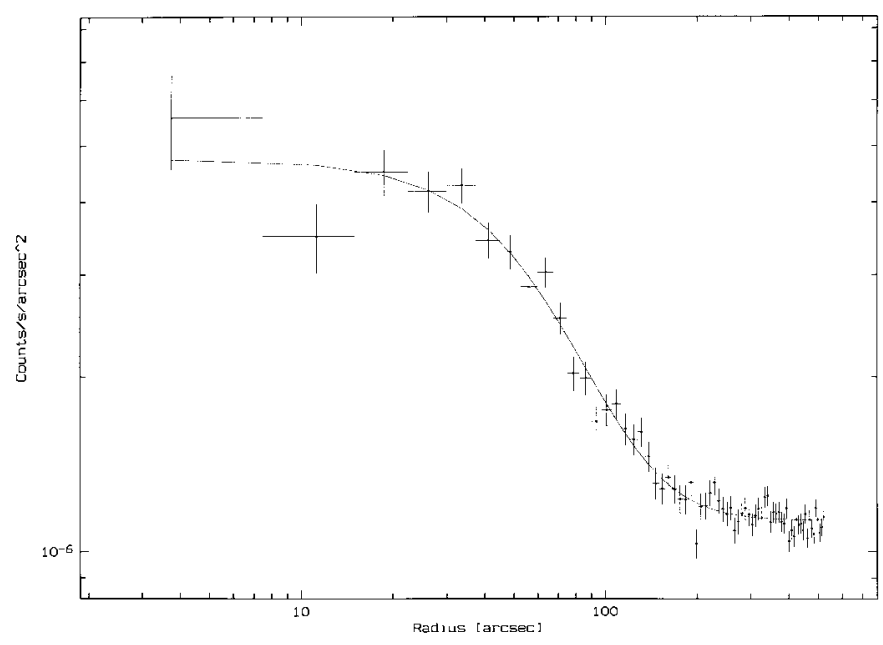

Fig. 10. RXJ1314-25: circularly averaged surface brightness profile in the [0.1-2.4] keV energy band. Bin size is $7.5^{\prime \prime}$ and the solid line is the King profile fit (Eq. (1)). The deviation of the inner two points from the model is due to the irregular morphology in the cluster centre.

Table 8. $\beta$-profile best-fit parameters with the corresponding 95\% confidence intervals.

\begin{tabular}{lcc}
\hline \hline Parameter & Abell 1451 & RXJ1314-25 \\
\hline$r_{\mathrm{c}}(\operatorname{arcsec})$ & $59 \pm 20$ & $81 \pm 28$ \\
$r_{\mathrm{c}}(\mathrm{kpc})$ & $240 \pm 90$ & $400 \pm 140$ \\
$\beta$ & $0.50 \pm 0.08$ & $0.77 \pm 0.23$ \\
\hline
\end{tabular}

that it may also be in the final stage of establishing equilibrium after a merger event.

Support for the merger scenario comes from different morphological and physical reasons which are summarized below:

- There is no single dominant galaxy. The brightest cluster galaxy (\#34, Table ??) is $35^{\prime \prime}$ away from the X-ray centroid, and $580 \mathrm{~km} \mathrm{~s}^{-1}$ from the cluster mean redshift.

The putative identification for the central radio source
Table 9. X-ray data for both clusters. $N_{\mathrm{H}}$ is taken from Dickey \& Lockman (1990). ASCA data for $T_{\mathrm{X}}$ and $L_{\mathrm{X}}$ in the [2-10] keV band (Matsumoto et al. 2001) are indicated correspondingly. The count-rate $C$ is in [0.1-2.4] keV band. The luminosities in [0.5-2], [2-10] keV and bolometric bands were obtained by extrapolation of the King profile out to $5^{\prime}$.

\begin{tabular}{lcc}
\hline \hline \multicolumn{1}{c}{ Parameter } & Abell 1451 & RXJ1314-25 \\
\hline$r_{\text {lim }}=5^{\prime}(\mathrm{Mpc})$ & 1.3 & 1.5 \\
$N_{\mathrm{H}}\left(10^{20} \mathrm{~cm}^{-2}\right)$ & 4.5 & 6.7 \\
$T_{\mathrm{X}}(\mathrm{keV}) \mathrm{ASCA}$ & $13.4_{-1.5}^{+1.9}$ & $8.7_{-0.6}^{+0.7}$ \\
Count-rate $C\left(r<r_{\text {lim }}\right)\left(\mathrm{cts} \mathrm{s}^{-1}\right)$ & 0.126 & 0.083 \\
$F_{\mathrm{X}}[0.5-2] \mathrm{keV}\left(10^{-12} \mathrm{erg} \mathrm{cm}^{-2} \mathrm{~s}^{-1}\right)$ & 4.4 & 3.2 \\
$F_{\mathrm{X}}[2-10] \mathrm{keV}\left(10^{-12} \mathrm{erg} \mathrm{cm}^{-2} \mathrm{~s}^{-1}\right)$ & 11.2 & 6.8 \\
$L_{\mathrm{X}}[0.5-2] \mathrm{keV}\left(10^{44} \mathrm{erg} \mathrm{s}^{-1}\right)$ & 6.8 & 7.4 \\
$L_{\mathrm{X}}[2-10] \mathrm{keV}\left(10^{44} \mathrm{erg} \mathrm{s}^{-1}\right)$ & 17.2 & 16.0 \\
$L_{\mathrm{X}}[2-10] \mathrm{keV}\left(10^{44} \mathrm{erg} \mathrm{s}^{-1}\right) \mathrm{ASCA}$ & $15.0^{\dagger}$ & $18.0^{\ddagger}$ \\
$L_{\mathrm{bol}}\left(10^{44} \mathrm{erg} \mathrm{s}^{-1}\right)$ & 39.8 & 34.0 \\
$n_{p}(0)\left(10^{-3} \mathrm{~cm}^{-3}\right)$ & 5.09 & 5.31 \\
$M_{\text {tot }}\left(<r_{\text {lim }}\right)\left(10^{14} M_{\odot}\right)^{*}$ & 8.6 & 9.7 \\
$M_{\text {gas }}\left(<r_{\text {lim }}\right)\left(10^{14} M_{\odot}\right)^{*}$ & 2.2 & 2.6 \\
$M_{\text {gas }} / M_{\text {tot }}\left(<r_{\text {lim }}\right)$ & 0.25 & 0.27 \\
\end{tabular}

ASCA/GIS luminosity out to 4 ', including the contribution from the QSO 1'30" south of the centre.

$¥$ ASCA/GIS luminosity out to $4^{\prime}$, including the contribution from the Sy1 galaxy $\sim 3^{\prime} 15^{\prime \prime}$ south-east of the centre.

* Assuming hydrostatic equilibrium.

Table 10. Details of the ATCA radio observations of the two clusters.

\begin{tabular}{cccccr}
\hline \hline & & & \multicolumn{3}{c}{ Restoring Beam } \\
Cluster & Frequency & RMS Noise & $b_{\text {maj }}$ & $b_{\text {min }}$ & PA \\
(Obs. Date) & (GHz) & mJy/beam & $\left({ }^{\prime \prime}\right)$ & $\left({ }^{\prime \prime}\right)$ & $(\mathrm{deg})$ \\
\hline Abell 1451 & 1.384 & 0.08 & 27.8 & 9.4 & -0.5 \\
(1999 Feb. 25) & 2.496 & 0.06 & 15.4 & 5.2 & -0.5 \\
RXJ1314-25 & 1.384 & 0.09 & 23.8 & 9.4 & 0.9 \\
$(1999$ Feb. 26) & 2.496 & 0.06 & 13.2 & 5.2 & 0.8 \\
\hline
\end{tabular}

(\#40, Table ??) is offset by $1300 \mathrm{~km} \mathrm{~s}^{-1}$ from the cluster mean redshift;

- Nearly regular X-ray emission, without substructures but slightly twisted inner part;

- No cooling flow region;

- Deviations from the observed scaling relations $L_{X}-T$ and $\sigma_{\mathrm{v}}-T$ (Xue \& Wu 2000), shown in Table 13. The cluster is significantly less luminous than expected from its measured temperature. The very high observed temperature $(13.4 \mathrm{keV})$ is probably an indication of a shock that occurred in the recent past. We cannot exclude a possible overestimation of the published ASCA temperature due to the presence of the background X-ray point source. However, the small difference (in the wrong sense) between the ASCA luminosity and our estimate with the QSO emission excluded (see Table 9) seems unlikely to account for a temperature overestimate of more than $4 \mathrm{keV}$. A new and more accurate measurement of the temperature is clearly needed; 
Table 11. Radio sources for Abell 1451 and RXJ1314-25 within 1 Abell radius of the cluster centre. Source coordinates are from VSAD. The peak $\left(S_{\text {peak }}\right)$ and integrated $\left(S_{\text {int }}\right)$ flux densities were measured using either VSAD or kview as described in the text. For Abell 1451 we adopt the X-ray emission peak as the centre.

\begin{tabular}{|c|c|c|c|c|c|}
\hline$N$ & \multicolumn{2}{|c|}{$(\mathrm{J} 2000)$} & $\begin{array}{c}v \\
\mathrm{GHz}\end{array}$ & $\begin{array}{l}S_{\text {peak }}(\mathrm{err}) \\
\mathrm{mJy} / \mathrm{bm}\end{array}$ & $\begin{array}{l}S_{\text {int }}(\mathrm{err}) \\
\mathrm{mJy}\end{array}$ \\
\hline \multicolumn{6}{|c|}{ Abell 1451} \\
\hline \multirow[t]{2}{*}{ R1 } & $12: 02: 51.67(0.04)$ & $-21: 26: 35.8(1.0)$ & 1.384 & 3.9 & 12.1 \\
\hline & & & 2.496 & 1.6 & 9.6 \\
\hline \multirow[t]{2}{*}{$\mathrm{R} 2$} & 12:02:56.73(0.06) & $-21: 28: 46.7(1.9)$ & 1.384 & $0.60(0.06)$ & $1.10(0.19)$ \\
\hline & & & 2.496 & - & - \\
\hline \multirow[t]{2}{*}{ R3 } & 12:02:58.94(0.04) & $-21: 38: 35.8(0.5)$ & 1.384 & $25.8(0.08)$ & $28.3(0.15)$ \\
\hline & & & 2.496 & $12.2(0.06)$ & 14.4( \\
\hline \multirow[t]{2}{*}{ R4 } & 12:03:06.66(0.05) & $-21: 39: 29.8(0.5)$ & 1.384 & $18.6(0.08)$ & $18.6(($ \\
\hline & & & 2.496 & $7.80(0.06)$ & 8.27 \\
\hline \multirow[t]{2}{*}{ R5 } & 12:03:08.65(0.05) & $-21: 39: 40.6(0.6)$ & 1.384 & $3.75(0.08)$ & 4.24 \\
\hline & & & 2.496 & 1.95 & 2.3 \\
\hline \multirow[t]{2}{*}{ R6 } & 12:03:10.59(0.04) & $-21: 29: 54.4(0.7)$ & 1.384 & $1.90(0.08)$ & 1.97 \\
\hline & & & 2.496 & 0.98( & 1.02( \\
\hline \multirow[t]{2}{*}{ R7 } & 12:03:17.35(0.04) & $-21: 32: 31.3(0.5)$ & 1.384 & $11.4(0.07)$ & 15.4 \\
\hline & & & 2.496 & $5.51(0.06)$ & 7.2 \\
\hline \multirow[t]{2}{*}{ R8 } & $12: 03: 26.97(0.04)$ & $-21: 30: 49.9(0.7)$ & 1.384 & $2.21(0.07)$ & $2.65(0.15)$ \\
\hline & & & 2.496 & $1.19(0.06)$ & 1.36 \\
\hline \multirow[t]{2}{*}{ R9 } & $12: 03: 32.53(0.04)$ & $-21: 33: 09.1(0.5)$ & 1.384 & $6.11(0.07)$ & 9.7 \\
\hline & & & 2.496 & $2.56(0.04)$ & 6.2 \\
\hline \multirow[t]{2}{*}{ R10 } & $12: 03: 32.85(0.05)$ & $-21: 36: 26.2(1.6)$ & 1.384 & $0.73(0.08)$ & $0.73(0.10)$ \\
\hline & & & 2.496 & $0.36(0.06)$ & \\
\hline \multirow[t]{2}{*}{ R11 } & $12: 03: 33.84(0.05)$ & $-21: 30: 22.1(1.7)$ & 1.384 & $0.39(0.08)$ & $0.36(0.13)$ \\
\hline & & & 2.496 & $0.30(0.05)$ & $0.47(0.13)$ \\
\hline \multirow[t]{2}{*}{ R12 } & 12:03:45.47(0.07) & $-21: 36: 11.7(2.1)$ & 1.384 & $0.52(0.07)$ & $0.80(0.17)$ \\
\hline & & & 2.496 & - & - \\
\hline \multirow[t]{2}{*}{ R13 } & 12:03:47.24(0.05) & $-21: 36: 15.1(1.3)$ & 1.384 & $0.78(0.07)$ & $1.01(0.16)$ \\
\hline & & & 2.496 & $0.31(0.05)$ & $1.09(0.19)$ \\
\hline \multirow[t]{3}{*}{ R14 } & 12:03:47.91(0.04) & $-21: 28: 33.1(0.5)$ & 1.384 & $5.53(0.08)$ & $5.32(0.14)$ \\
\hline & & & 2.496 & $2.70(0.06)$ & $2.79(0.12)$ \\
\hline & & RXJ1314-2 & & & \\
\hline \multirow[t]{2}{*}{ R1 } & 13:14:00.90(0.04) & $-25: 16: 53.7(0.7)$ & 1.384 & $2.09(0.09)$ & $2.38(0.1)$ \\
\hline & & & 2.496 & $0.84(0.06)$ & $1.13(0.1)$ \\
\hline \multirow[t]{2}{*}{$\mathrm{R} 2$} & 13:14:18.62(0.05) & $-25: 15: 47.0(1.0)$ & 1.384 & $1.16(0.03)$ & 13.0 \\
\hline & & & 2.496 & - & - \\
\hline \multirow[t]{2}{*}{ R3 } & 13:14:30.31(0.04) & $-25: 17: 14.2(0.7)$ & 1.384 & $1.93(0.08)$ & 4.4 \\
\hline & & & 2.496 & $0.54(0.04)$ & 1.7 \\
\hline \multirow[t]{2}{*}{ R4 } & $13: 14: 34.31(0.06)$ & $-25: 11: 59.9(1.6)$ & 1.384 & $0.69(0.09)$ & $0.64(0.2)$ \\
\hline & & & 2.496 & $0.62(0.07)$ & $0.57(0.1)$ \\
\hline \multirow[t]{2}{*}{ R5 } & $13: 14: 45.90(0.05)$ & $-25: 15: 05.5(0.7)$ & 1.384 & $1.57(0.04)$ & 6.8 \\
\hline & & & 2.496 & $0.48(0.04)$ & 1.5 \\
\hline \multirow[t]{2}{*}{ R6 } & 13:14:48.64(0.04) & $-25: 16: 18.3(0.6)$ & 1.384 & $3.20(0.09)$ & $3.65(0.2)$ \\
\hline & & & 2.496 & $1.47(0.05)$ & $2.20(0.1)$ \\
\hline
\end{tabular}

- Based on numerical simulations (Roettiger et al. 1997; Belsole et al. 2002), the regular X-ray morphology and high $\mathrm{X}$-ray temperature are fully compatible with expectations from a past merger.

We can also determine the dynamical status of the cluster by comparing its kinetic and potential energies. From the measured velocity dispersion we find $\beta_{\mathrm{spec}}=\mu m_{\mathrm{p}} \sigma_{\mathrm{v}}^{2} / k T=$ $0.84 \pm 0.25$, while from the X-ray emission, with the correction factor from Bahcall \& Lubin (1994), we have $\beta_{\mathrm{X}}^{c}=1.25 \beta_{\mathrm{X}}=$ $0.63 \pm 0.1$. These values are consistent within the uncertainties, indicating that the gas and galaxy motions are close to equipartition.
Table 12. Radio-optical identifications in Abell 1451 and RXJ1314-25. $N$ refers to the identification number given in Table 11 and $\Delta \mathrm{r}$ is the radius-vector offset between radio and SuperCOSMOS optical positions. T refers to the SuperCOSMOS image classification: 1 = galaxy; 2 = star.

\begin{tabular}{rccccc}
\hline \hline$N$ & $\begin{array}{c}\text { RA } \\
\text { Optical }\end{array}$ (J2000) & $\begin{array}{c}B_{J} \\
(\mathrm{mag})\end{array}$ & $\begin{array}{c}\Delta r \\
\left({ }^{\prime \prime}\right)\end{array}$ & $T$ \\
\hline \multicolumn{5}{c}{ Abell 1451 } \\
R1 & $12: 02: 51.56$ & $-21: 26: 35.4$ & 19.26 & 1.6 & 1 \\
R2 & $12: 02: 56.81$ & $-21: 28: 55.8$ & 18.42 & 9.1 & 1 \\
R3 & $12: 02: 58.92$ & $-21: 38: 36.4$ & 18.18 & 0.7 & 1 \\
R5 & $12: 03: 08.68$ & $-21: 39: 40.0$ & 20.12 & 0.7 & 2 \\
R6 & $12: 03: 10.35$ & $-21: 29: 54.2$ & 19.13 & 3.4 & 1 \\
R7 & $12: 03: 17.47$ & $-21: 32.27 .4$ & 19.41 & 4.2 & 1 \\
R8 & $12: 03: 26.98$ & $-21: 30: 51.5$ & 18.19 & 1.6 & 1 \\
R11 & $12: 03: 45.70$ & $-21: 36: 11.9$ & 18.98 & 3.5 & 1 \\
R12 & $12: 03: 47.14$ & $-21: 36: 12.8$ & 20.28 & 2.8 & 1 \\
R13 & $12: 03: 48.11$ & $-21: 28: 33.1$ & 20.40 & 3.2 & 2 \\
& & & & & \\
R1 & $13: 14: 00.89$ & $-25: 16: 54.3$ & 19.81 & 0.6 & 2 \\
R2 & $13: 14: 23.78$ & $-25: 07: 51.8$ & 21.07 & 2.9 & 1 \\
R3 & $13: 14: 30.36$ & $-25: 17: 17.4$ & 21.40 & 3.3 & 1 \\
R4 & $13: 14: 34.27$ & $-25: 11: 58.9$ & 20.07 & 1.1 & 1 \\
R5 & $13: 14: 46.17$ & $-25: 15: 09.1$ & 21.37 & 5.1 & 1 \\
\hline
\end{tabular}

Table 13. Scaling relations for Abell 1451 and RXJ1314-25. $\sigma_{\mathrm{v}}-T$ is from Xue \& Wu (2000), while $L_{\mathrm{bol}}-T$ is from Arnaud \& Evrard (1999). The temperature from ASCA is in $\mathrm{keV}, L_{\mathrm{bol}}$ in units of $10^{44} \mathrm{erg} \mathrm{s}^{-1}$ and $\sigma_{v}$ in $\mathrm{km} \mathrm{s}^{-1}$ are from this paper.

\begin{tabular}{lrcccc}
\hline \hline Cluster & $\begin{array}{c}T \\
(\text { obs })\end{array}$ & $\begin{array}{c}\sigma_{\mathrm{v}} \\
(\mathrm{obs})\end{array}$ & $\begin{array}{c}\sigma_{\mathrm{v}} \\
\left(\sigma_{\mathrm{v}}-T\right)\end{array}$ & $\begin{array}{c}L_{\text {bol }} \\
(\text { obs })\end{array}$ & $\begin{array}{c}L_{\text {bol }} \\
\left(L_{\text {bol }}-T\right)\end{array}$ \\
\hline Abell 1451 & 13.4 & 1330 & 1670 & 39.8 & 116 \\
RXJ1314-25 & 8.7 & 1100 & 1261 & 34.0 & 33.5 \\
\hline
\end{tabular}

If a merger occurred recently we might expect signatures at radio wavelengths, such as radio halo/relic sources, and possibly tailed sources (e.g. Enßlin et al. 1998; Reid et al. 1998; Röttgering et al. 1994). There is no evidence for a radio halo, although there is a tailed source (R7) near the cluster centre which could have disrupted it (Giovannini 1999; Liang et al. 2000).

\section{2. $R X J 1314-25$}

RXJ1314-25 is morphologically and dynamically very different from Abell 1451. It shows a clear bi-modal structure - there are two groups in velocity space separated by $\sim 1700 \mathrm{~km} \mathrm{~s}^{-1}$ (cf. Table 6 and Fig. 5) which are also separated in the projected galaxy distribution (cf. Fig. 6). The dominant galaxies of each group are separated by $\sim 1000 \mathrm{~km} \mathrm{~s}^{-1}$ in redshift space, and $2^{\prime} 25^{\prime \prime}$, or $\approx 700 \mathrm{kpc}$, in projected distance.

The X-ray emission is elongated, with the centroid located between the two dominant galaxies. The elongation, however, is rotated by $\approx 20^{\circ}$ from the axis connecting the two BCGs. This may simply be due to the decoupling between the galaxies and gas during the merger. 
There are no cluster radio sources within the X-ray extension, with the possible exception of the weak (uncatalogued) source at the position of galaxy \#48. If we are witnessing an interaction between two sub-clusters, we might expect stronger radio activity than observed. However, residual sidelobes from a strong background source $\sim 7^{\prime}$ south of the centre hamper the detection of any very extended emission. In addition, a more compact ATCA antenna configuration is needed to improve sensitivity to low surface brightness emission. There are, however, two extended radio sources, one of which (R2) has a steep radio spectrum and no optical counterpart, and is therefore a plausible candidate for a relic source.

The observed $L_{\mathrm{X}}, T$ and $\sigma_{\mathrm{v}}$ for RXJ1314-25 are in good agreement with the $L_{\mathrm{X}}-T$ and $\sigma_{\mathrm{v}}-T$ scaling relationships (Table 13), suggesting that the merger has progressed to the stage where the transient shock heating and radio activity have dissipated. On the other hand, if the cluster is in a pre-merging phase, then it is unusual that the X-ray elongation is not aligned with the group centres and that there is no sign of X-ray substructure around the eastern group, as revealed, for example, in numerical simulations (Roettiger et al. 1997; Takizawa 2000). The scattered appearance of the projected galaxy distribution of the eastern group compared to the western group (Fig. 6) also supports a post-merging scenario.

In conclusion, our observations suggest that neither cluster is relaxed following a recent merger. However, their properties and scaling laws are quite different, illustrating the diversity in the merging and relaxation processes in cluster formation and evolution. The current data for the two clusters are compatible with the expectations from the merger of a small group with a bigger cluster for Abell 1451, and nearly equal mass groups for RXJ1314-25. Deep XMM and Chandra observations, coupled with detailed numerical simulations are needed to assess these hypotheses and better understand the many aspects of the physical processes occurring during accretion and relaxation over the course of a cluster merger.

Acknowledgements. We would like to thank Romain Teyssier, John Hughes and Pierre-Alain Duc for numerous discussions about simulations, data reduction and analysis. We are especially indebted to Hector Flores and Dario Fadda for providing us with the observation of galaxy \#48 in RXJ1314-25 (CFHT, June 2001). We thank the referee Reinaldo de Carvalho for valuable comments.

\section{References}

Abell, G. O. 1958, ApJS, 3, 211

Abell, G. O., Corwin, H. G., \& Olowin, R. P. 1989, ApJS, 70, 1

Arnaud, M., \& Evrard, A. 1999, MNRAS, 305, 631

Ashman, K. M., Bird, K. M., \& Zepf, S. E. 1994, AJ, 108, 2348

Bahcall, N., \& Lubin, L. 1994, ApJ, 426, 513

Baier, F. W., Lima Neto, G. B., Wipper, H., \& Braun, M. 1996, AN, 317,77

Bautz, L. P., \& Morgan, W. W. 1970, ApJ, 162, L149

Beers, T. C., Flynn, K., \& Gebhardt, K. 1990, AJ, 100, 32 (ROSTAT)

Belsole, E., Sauvageot, J.-L., \& Teyssier, R. 2002 [astro-ph/0203297]

Cavaliere, A., \& Fusco-Femiano, R. 1976, A\&A, 49, 137

Condon, J. J. 1997, PASP, 109, 166

Donahue, M., Voit, G. M., Gioia, I., et al. 1998, ApJ, 502, 550
D’Agostino, R. B., \& Stephens, M. A. 1986, Goodness-of-fit Techniques (Marcel Dekker, New York)

Maddox, G., Sutherland, W. J., Efstathiou, G., \& Loveday, J. 1990b, MNRAS, 243, 692

Danese, L., De Zotti, G., \& di Tullio, G. 1980, A\&A, 82, 322

Dickey, J. M., \& Lockman, F. J. 1990, ARA\&A, 28, 215

Dressler, A., \& Shectman, S. A. 1988, AJ, 95, 985

Efron, B., \& Tibshirani, R. 1986, Stat. Sci., 1, 54

Enßlin, T. A., Biermann, P. L., Klein, U., \& Kohle, S. 1998, A\&A, 332,395

Fabian, A. C. 1994, ARA\&A, 32, 277

Geller, M. J., \& Beers, T. C. 1982, PASP, 94, 421

Giovannini, G. 1999, in Diffuse thermal and relativistic plasma in galaxy clusters, ed. H. Böhringer, L. Feretti, \& P. Schuecker, MPE Rep., 271, 13

Girardi, M., Fadda, D., Giuricin, G., et al. 1996, ApJ, 457, 61

Gómez, P. L., Hughes, J. P., \& Birkinshaw, M. 2000, ApJ, 540, 726

Gooch, R. 1996, PASA, 14(1), 106

Hambly, N. C., MacGillivray, H. T., Read, M. A., et al. 2001, MNRAS, 326,1279

Hartigan, P. M. 1985, Appl. Stat., 34, 3

Hughes, J. P., Yamashita, K., Okumura, Y., et al. 1988, ApJ, 327, 615

Harrison, E. R. 1974, ApJ, 191, L51

King, I. R. 1962, AJ, 67, 471

Kurtz, M. J., \& Mink, D. J. 1998, PASP, 110, 934

Liang, H., Hunstead, R., Birkinshaw, M., \& Andreani, P. 2000, ApJ, 544,686

Lémonon, L., Pierre, M., Hunstead, R., et al. 1997, A\&A, 326, 34

Lumsden, S. L., Collins, C. A., Nichol, R. C., Eke, V. R., \& Guzzo, L. 1997, MNRAS, 290, 119

Matsumoto, H., Pierre, M., Tsuru, T. G., \& Davis, D. 2001, A\&A, 374,28

Pierre, M., Böhringer, H., Ebeling, H., et al. 1994, A\&A, 290, 725

Pierre, M., Oukbir, J., Dubreuil, D., et al. 1997, A\&AS, 124, 283

Pierre, M., \& Starck, J.-L. 1998, A\&A, 330, 801

Pisani, A. 1996, MNRAS, 278, 697

Raymond, J. C., \& Smith, B. W. 1977, ApJS, 35, 419

Reid, A. D., Hunstead, R. W., \& Pierre, M. M. 1998, MNRAS, 296, 531

Rizza, E., Loken, C., Bliton, M., et al. 2000, AJ, 119, 21

Roettiger, K., Loken, C., \& Burns, J. O. 1997, ApJS, 109, 307

Röttgering, H., Snellen, I., Miley, G., et al. 1994, ApJ, 436, 654

Sarazin, C. L. 1986, Rev. Mod. Phys., 58, 1

Sault, R. J., Teuban, P. J., \& Wright, M. C. H. 1995, in Astronomical Data Analysis Software and Systems IV, ed. R. A. Shaw, H. E. Payne, \& J. J. E. Hayes (San Francisco: ASP), ASP Conf. Ser., 77, 433

Schoenmakers, A. P., de Bruyn, A. G., Röttgering, H. J. A., \& van der Laan, H. 2001, A\&A, 374, 861

Silverman, B. 1986, Density Estimation for Data Analysis and Statistics (London: Chapman \& Hall)

Starck, J.-L., \& Pierre, M. 1998, A\&AS, 128, 397

Takizawa, M. 2000, ApJ, 532, 183

Tonry, J., \& Davis, M. 1979, AJ, 84, 1511

Valtchanov, I. 1999, in Astronomical Data Analysis Software and Systems VIII, ed. D. M. Mehringer, R. L. Plante, \& D. A. Roberts (San Francisco: ASP), ASP Conf. Ser., 172, 38

Voges, W., Aschenbach, B., Boller, Th., et al. 1999, A\&A, 349, 389

West, M. J. 1994, In Proc. XIV Moriond Astrophysics Meeting, Clusters of Galaxies, ed. F. Durret, A. Mazure, \& J. Trân Than Vân (Gif-sur-Yvette: Éditions Frontières), p. 23

Xue, Y.-J., \& Wu, X.-P. 2000, ApJ, 538, 65

Yahil, A., \& Vidal, N. V. 1977, ApJ, 214, 347

Zimmermann, H. U., Becker, T., Belloni, T., et al. 1994, EXSAS User's Guide 